\title{
Correlation between Cosmic Ray muons and atmospheric temperature in the lower stratosphere
}

\author{
Maghrabi A.H ${ }^{1}$ \\ National Centre for Applied Physics, King Abdulaziz City for Science and Technology, \\ Address, P.O.Box 6086Riyadh 11442, Saudi Arabia. \\ Email:amaghrabi@kacst.edu.sa
}

\section{Al Mutairi M.M}

National Centre for Applied Physics, King Abdulaziz City for Science and Technology, Address, P.O.Box 6086Riyadh 11442, Saudi Arabia.

Email:almuatairi@kacst.edu.sa

\begin{abstract}
In this study, the relationship between cosmic ray muons and the atmospheric temperature in the lower stratosphere has been investigated and established. Cosmic ray data from the KACST muon detector $(\mathrm{Rc}=14.4 \mathrm{GV})$ and radiosonde measurements from Riyadh airport, for the period 2002-2012, have been used for this purpose. We have found that the temperature in the lower stratosphere is correlated with the cosmic ray muons detected at ground level. The results obtained are in agreement with previous studies. The technique was then used to predict temperature variations in the lower stratosphere. It was found that the temperature can be reproduced with a mean bias error of $0.5{ }^{\circ} \mathrm{C}$ and a root mean square error of $3{ }^{\circ} \mathrm{C}$.
\end{abstract}




\section{Introduction}

Primary cosmic ray particles (mainly protons) collide with atmospheric molecules, producing a cascade of secondary particles. These secondaries are affected by variations in the atmosphere before reaching detectors. Cosmic ray muons form the majority of the detected particles at ground and underground levels[1]. The amount of detected muons is mainly dependant on the atmospheric mass above the detector and on temperature changes [2]. The atmospheric mass is represented by the atmospheric pressure at ground level and can be easily obtained by correlating the observed number of cosmic rays with the atmospheric pressure at the observation level[3]. On the other hand, the temperature corrections are slightly more complicated and several methods have been suggested to account for them [e.g., 1 and 3]. These include correlation of the muon rates with one of the following parameters: the atmospheric weighted temperature [4-5], the temperature at ground level [3 and 6], or with the temperature at the level of pion production [7-11].

The lower stratosphere is approximately defined to be the level in the atmosphere with pressure between $70 \mathrm{mb}$ and $200 \mathrm{mb}$. At these heights, an interaction takes place between the primary cosmic ray particles and atmospheric molecules, and the parent pions are produced. These pions, which have very short life times, decay into different subatomic particles, including muons[1 and 9]. In this study the correlation between the cosmic ray muons observed by the King Abdulaziz City for Science and Technology (KACST) detector, and the temperature at the lower stratosphere, will be investigated. The temperature coefficient from this correlation will be used to remove the effect of the temperature of the atmosphere from the detected muons. Then, with aid of the obtained coefficient, the correction formula will be inverted to calculate the atmospheric temperature in the lower stratosphere using the muon observations. The predictability of this approach will be tested and validated.

\section{Instrumentation and Methods}

Cosmic ray measurements began in 2002, when KACST installed the first muon detector in the country in Riyadh (lat. 24 43; long. 46 40; alt. 613 m; cutoff rigidity 14.4 GV), Saudi Arabia. This is a single channel scintillator detector built in collaboration with the University of Adelaide, Australia, which is designed for long term cosmic ray variation studies. The technical details of this detector can be found elsewhere [12-13]. The detector is a $1 \mathrm{~m}^{2}$ plastic scintillator viewed by a Hamamatsu H8711 photomultiplier tube (PMT), and both are placed in light tight box. As a cosmic ray muon strikes the scintillator the scintillator materials will emit flashes of fluorescent light. The light produced is detected by the PMT and converted to electrical signals, after removal of the background noise. The selected signals then undergo amplification processes using home-made electronic circuits [13]. An Analogue to Digital Converter (ADC) is used to digitise the signals and send them to a PC card. The data are acquired every 15 minutes with a sampling frequency of $1 \mathrm{~s}$ using software developed in-house.

Twice daily radiosonde data obtained from Saudi Presidency of Meteorological Environment (PME), for the period between 2002 and 2012, have been used to extract the temperature between $70 \mathrm{mb}$ and $200 \mathrm{mb}$. The average temperature values between $70 \mathrm{mb}$ and $200 \mathrm{mb}$ have been used in this study. 
The relative variations of cosmic ray muons due to the effects of atmospheric pressure and temperature is described as [7-9]:

$\frac{\mathrm{I}-\mathrm{I}_{0}}{\mathrm{I}_{0}}=\alpha\left(\mathrm{P}-\mathrm{P}_{0}\right)+\beta\left(\mathrm{T}-\mathrm{T}_{0}\right)$

Here, $I_{0}, P_{0}$, and $T_{0}$ are the mean values of muon rate, ground level pressure, and the temperature between $70 \mathrm{mb}$ and $200 \mathrm{mb}$ during the study period. The pressure and temperature coefficients are $\alpha$ and $\beta$, respectively, which must be obtained from regression analyses.

\section{Results and Discussions}

\subsection{Pressure and Temperature Effects}

Figure 1 displays the yearly variations of the monthly averages of (a) the raw muon rate, (b) atmospheric pressure, and (c) the temperature stratospheric temperature. Clear seasonal variations are evident for the muon rate, with a maximum in summer time and a minimum during winter. On the other hand, the pressure and the temperature exhibited the opposite trend.
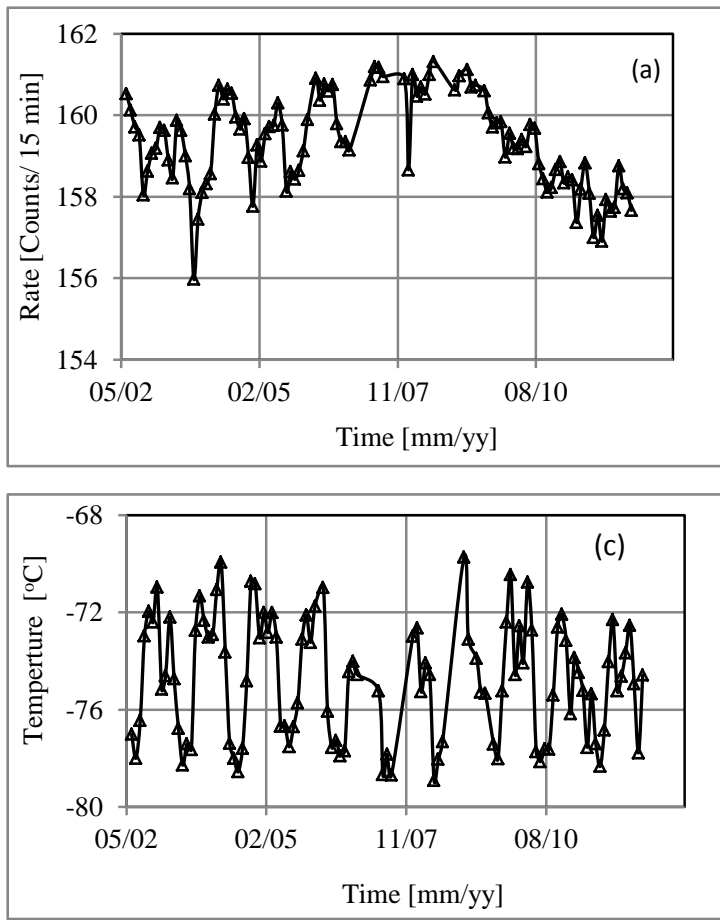

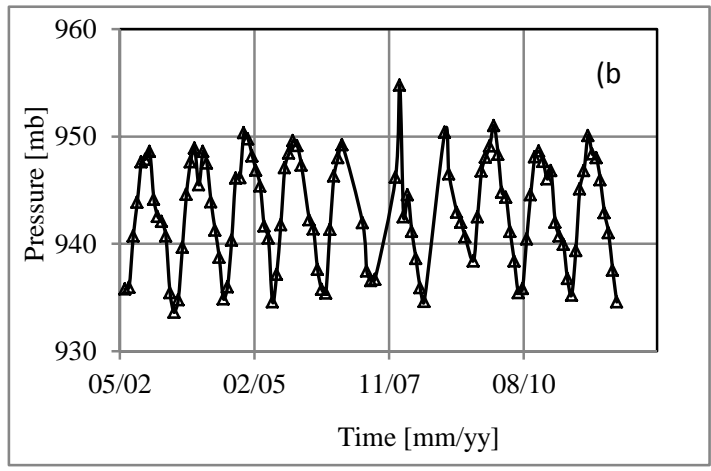

Figure 1: Timeseries of monthly means of the (a) raw muon rates, (b) atmospheric pressure, and (c) stratospheric temperature, during the 10 years of the study.

The pressure effect must be removed because it is the most important factor affecting the observed cosmic ray particles. The mean monthly values of atmospheric pressure were plotted against the raw muon rates for the whole dataset, as shown in Figure 2.

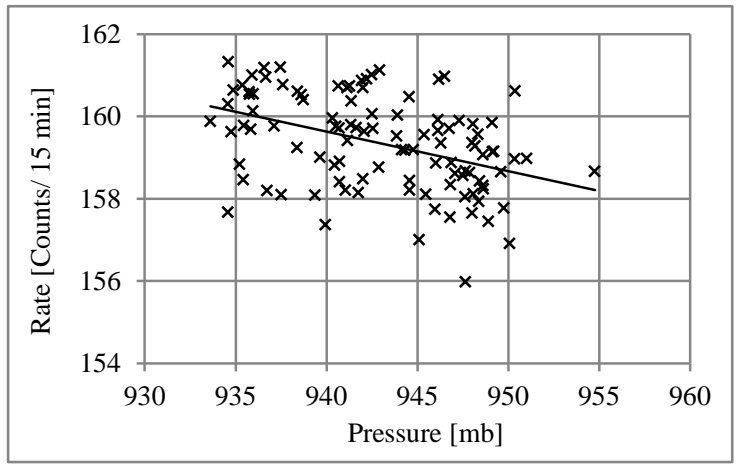

Figure 2: Scatterplot of the muon mean monthly values against atmospheric pressure. The solid line is the regression line.

While there was some scatter in the data, the anti-correlation between the two parameters is obvious. The pressure coefficient obtained from the least square fit between the two variables was of $-0.12 \pm 0.03 \% / \mathrm{mb}$. This 
coefficient was used to remove the pressure effect from the muon data as shown in Figure 3. Apparently, the pressure corrected data follow the opposite trend when compared with raw muon rates. The pressure effect on the cosmic ray muons has approximately a magnitude of about $1 \%$ in difference with the raw muon rates.

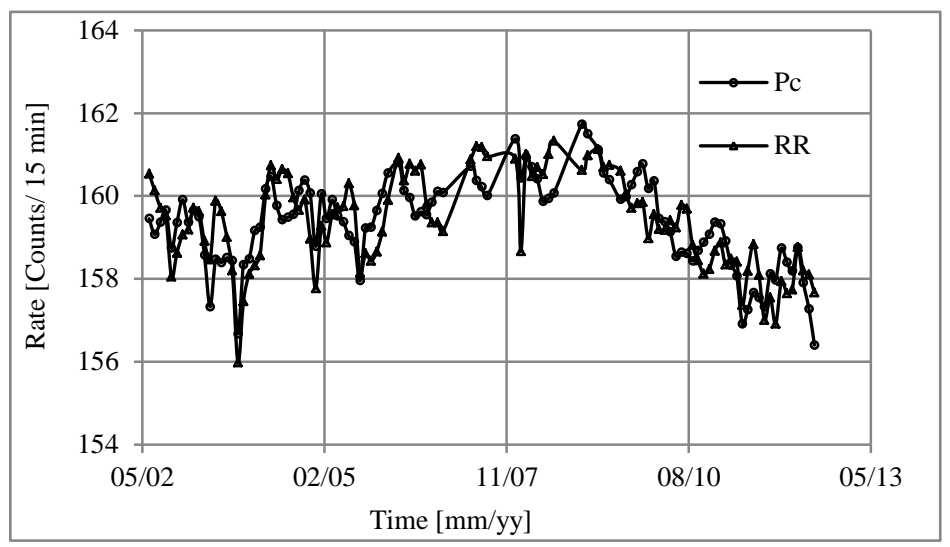

Figure 3: Monthly mean values of the corrected muon rate $(P c)$ compared with the raw rate $(R R)$.

The mean monthly pressure corrected data were plotted against stratospheric temperature as indicated in Figure 4.a. Figure 4.b is same as Figure 4.a, with the exception that the former shows the relationship between the twelve-month binned temperatures and the pressure corrected muon rates. While the scatter in the averaged data is reduced, it is obvious from both figures that the two variables are positively correlated with each other. Regression analyses between the two variables performed using the two datasets - the interannual based on monthly averages and the twelve-month binned means - give the same slope (temperature coefficient) of $0.10 \pm$ $0.024 \% / \mathrm{oC}$, and correlation coefficients of 0.75 for the former dataset, 0.41 for the latter.

The correlation between the two variables implies that the parent pions will have less probability of decay to produce muons before they interact as the temperature decreases (winter), while the opposite is the case during warm seasons (summer). This result is in agreement with those previously reported [7-9].
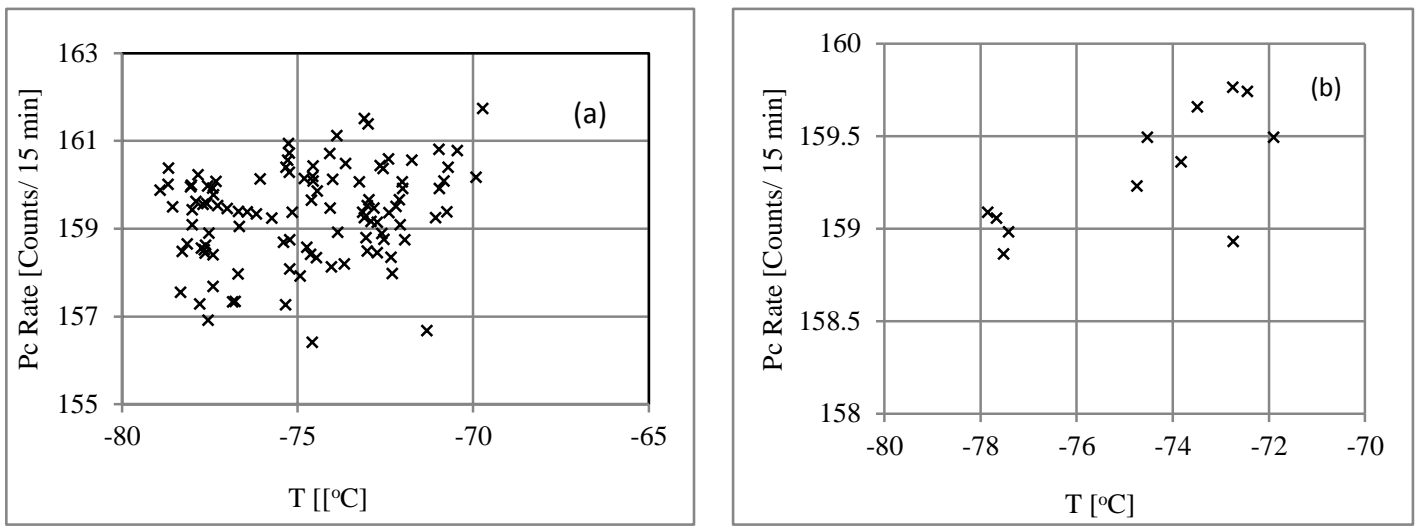

Figure 4: (a) Scatterplot of the monthly mean pressure corrected muon rate and the stratospheric temperature, $(b)$ the twelve-month binned temperature and pressure corrected muon rates are plotted against each other

The interannual variations of the mean monthly values of the raw and temperature corrected muon rates, for the considered time period, are presented in Figure 5. It can be seen that the temperature corrected rate follows the same trend as the raw muon rate. The difference in magnitude between the two rates is about $0.4 \%$. When the pressure (Figure 3 ) and temperature (Figure 5) effects are removed from the raw muon observations, seasonal variations are still observed. This implies that there are still some other terrestrial and extra-terrestrial factors which may need to be considered to account for this seasonality [14]. 


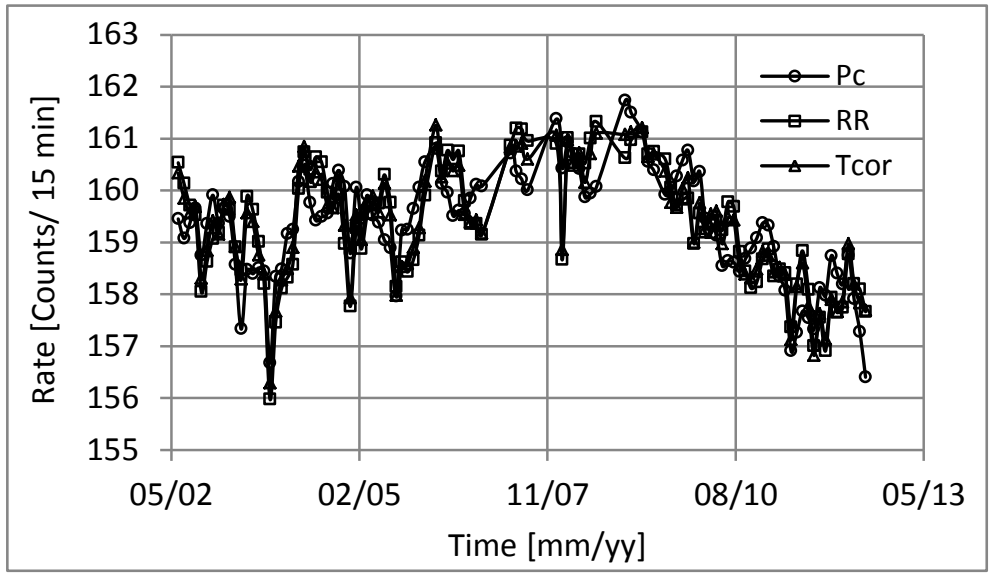

Figure 5: Comparison of the monthly values of the raw muon rates (RR)against those of the pressure corrected $(P c)$, and with the low stratosphere temperature correction applied (Tcor).

\subsection{Calculating the Stratospheric Temperature}

The correlation between the muon rates and the temperature in the lower stratosphere suggests the possibility of using the cosmic ray muons to predict temperature at that level of the atmosphere as suggested by [15-16]. Recall the second part of Equation (1):

$\frac{\mathrm{I}-\mathrm{I}_{0}}{\mathrm{I}_{0}}=\beta\left(\mathrm{T}-\mathrm{T}_{0}\right)$

By inverting this equation to:

$\mathrm{T}=\frac{1}{\beta}\left(\frac{\mathrm{I}-\mathrm{I}_{0}}{\mathrm{I}_{0}}\right)+\mathrm{T}_{0},(3)$

the stratospheric temperature can be calculated from the muon data at the detection level. To test this approach the dataset was divided into two groups. The first covers the period 20022006 (1148 daily values) and the second covers the period from 2007 to 2012 (335 daily values). The daily values of the pressure corrected muon rate and stratospheric temperature for the first period have been used to parameterise the relationship between the two variables, whereas the second dataset has been used to validate the method. The mean values of the temperature $\left(T_{o}\right)$, muon rate $\left(I_{o}\right)$, and temperature coefficient $(\beta)$, as well as the muon rates (I), have been used to calculate the temperature $(\mathrm{T})$ of the lower stratosphere using Equation (3). The statistical results of this approach for the first and second datasets are presented in the Table (1).

Table (1): Summary of mean, maximum, and minimum temperatures, and MBE and MPE for datasets 1

and 2.

\begin{tabular}{|l|r|r|r|r|r|r|r|r|}
\hline & $T$ & Predicted Set1 & MBE1 & MPE1 & TEMP & Predicted Set 2 & MBE2 & MPE2 \\
\hline Mean & $-74.86 \pm 3.24$ & $-77.31 \pm 5.1$ & 2.44 & 1.53 & $-75.33 \pm 3.0$ & $-75.73 \pm 3.5$ & 0.40 & 0.25 \\
\hline Minimum & -82.10 & -90.63 & -12.37 & -7.75 & -81.30 & -84.12 & -6.98 & -4.37 \\
\hline Maximum & -66.90 & -66.35 & 22.12 & 13.86 & -66.50 & -69.07 & 10.78 & 6.76 \\
\hline
\end{tabular}

measured temperature, $T_{\text {ical }}$ is the temperature predicted by the model. 
Figures 6.a and 6.b show the frequency distribution of the difference between the measured and predicted temperatures for the first and the second datasets, respectively. The mean bias errors (MBE) were $2.4{ }^{\circ} \mathrm{C}$ for the first dataset and $0.40{ }^{\circ} \mathrm{C}$ for the second. The mean percentage error (MPE) was $1.5 \%$, and the standard deviation was $4.6{ }^{\circ} \mathrm{C}$, for the first dataset. The predictability of the measured temperatures for the second dataset has MPE $=0.25 \%$ and standard deviation $=2.6{ }^{\circ} \mathrm{C}$. It is important to mention that the proposed method of predicting the temperature may under- or over-estimate the measured data in some situations. These cases may be due to specific atmospheric or extra-terrestrial disturbances at the time of observations, which may occur higher in the atmosphere during changes of the season. However, the overall results indicate that the proposed method has superior performance when calculating the lower stratospheric temperatures alongside knowledge of the measured muon data.
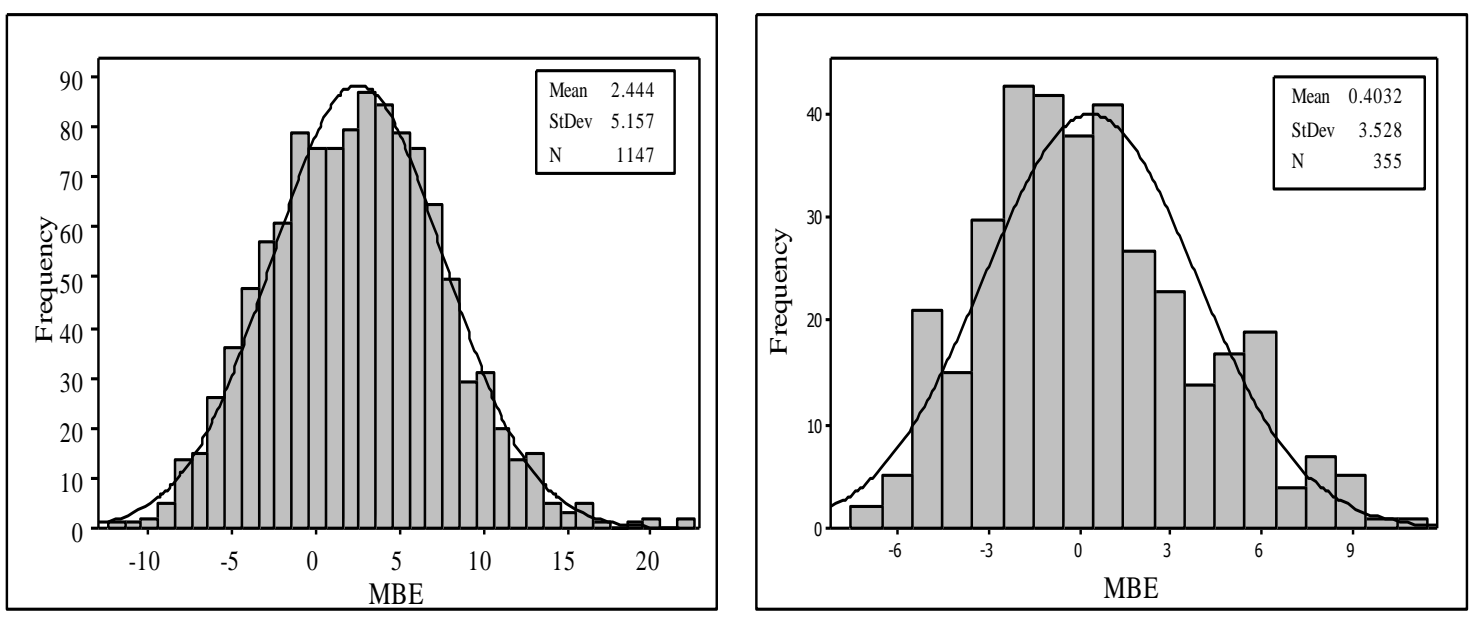

Figure 6: (a)Histogram showing the frequency distribution of the MBE (in degrees) of the measuredpredicted stratospheric temperature (dataset for the period 2002-2006) superimposed on the normal distribution curve, obtained using Equation (3).(b) same as Figure 6.a but for the dataset covering 20072012.

\section{Conclusions}

In this paper, the effect of the stratospheric temperature on the rate of cosmic ray muons has been investigated. Cosmic ray measurements from the KACST muon detector located in Riydah, Saudi Arabia (lat. 24 43; long. 46 40; alt. 613 m; cutoff rigidity 14.4 GV) over a ten year period, and coincident radiosonde data, have been used in this study. It was found that the muon rate is positively correlated with the stratospheric temperature. This means that during winter the parent pions have less probability to decay to produce muons, while the opposite will be the case during summer. This result is in agreement with previous studies. The correlations between the two variables were inverted to calculate the temperature in the lower stratosphere using knowledge of muon observations. The proposed technique provides excellent predictability for the stratospheric temperatures.

\section{Acknowledgments}

The authors would like to thank King Abdulaziz City for Science and Technology (KACST) for supporting this work. 


\section{References}

[1] L. Dorman, 2004, Cosmic rays in the Earth's atmosphere and underground, Kluwer Academic Publishers, USA.

[2] A. Maghrabi, et al., Influence of the Atmospheric Mass on the High Energy Cosmic Ray Muons during a Solar Cycle, Advances in Astronomy doi.org/10.1155/2015/939146; 2015.

[3] DeMendonça et al., Analysis of atmospheric pressure and temperature effects on cosmic ray measurements, J. Geophys. Res 118, 1403. 2013.

[4] V. .Dvornikov, et al., Determination of the mass-average temperature on the cosmic ray intensity data, Geomag. Aeron., 16 (5), 923-925, 1976.

[5] A. Maghrabi, A., et al., Atmospheric-weighted temperature and its influence on cosmic ray muons, 34rd -ICRC2015, The Hague, The Netherlands .

[6] A Maghrabi et al., The Ground Temperature Effect on Cosmic-Ray Muons at Mid latitude City, Proc.23d ECRS ,2014.

[7] A. Duperier, The meson intensity at the surface of the Earth and the temperature at the production level, Proc. Phys. Soc., 62A, 684-696, 1949.

[8] V. Yanke, et al. Temperature effect of general component seen by cosmic ray detectors, Proceedings of 32nd ICRC, 11, 377-380, 2011.

[9] C.Braga et al; Temperature effect correction for the cosmic ray muon data observed at the Brazilian Southern Space Observatory in São Martinho da Serra, 23rd ECRS and 32nd Russian Cosmic Ray Conference doi:10.1088/1742-6596/409/1/012138.

[10] A.Dmitrieva et al., Temperature effect correction for muon flux at the Earth surface: estimation of the accuracy of different methods, Journal of Physics: Conference Series 409, 012130, 2013.

[11] A. Maghrabi , Almutayri M. Atmospheric Effect on Cosmic Ray muons at High Cutoff Rigidity Station, Advances in Astronomy, V. 2016.

[12] A. Maghrabi , et al., The KACST muon detector and its application to cosmic-ray variations studies; Adv. Space Res.50 ,700. 2012,

[13] A. Maghrabi et al., On the calibration of a single channel cosmic ray particle detector, Proc. SPIE 9154, 91542K, 2014 .

[14] M.Ambrosio et al. , Seasonal variations in the underground muon intensity as seen by MACRO, Astroparticle Phys., 7(1-2), 109-124, 1997.

[15] M. Bertaina et al., The atmospheric muon flux in correlation with temperature variations in the low stratosphere (50-200 mb)., 30th ICRC, Mérida, Yucatán, Mexico, 2007,

[16] M . Aglietta., et al., Temperature variations in the low stratosphere (50-200 hPa) monitored by means of the atmospheric muon flux;IL NUOVO CIMENTO; 31 C, N. 22008. 